\title{
Synthesis of Neutral Medium Inhibitor and Study of its Inhibition Efficiency
}

\author{
Cheng PENG ${ }^{1, a^{*}}$, HuangXian-QIU ${ }^{1, b}$ and FuChao-YANG ${ }^{2, c}$ \\ ${ }^{1}$ Reasearch and Development Center of WISCO, Wuhan 430080 \\ ${ }^{2}$ School of Chemistry \& Chemical Engineering of Huazhong University of Science and Technology, \\ Wuhan 430074
}

a164348096@qq.com, bhuangxq@wisco.com.cn, ${ }^{c} c y f u @$ hust.edu.cn

\begin{abstract}
With fatty acid and amino acid as raw materials, the neutral medium inhibitor was synthesized. The inhibitory performance of corrosion inhibitor for corrosion of Q235 steel in neutral water medium was investigated by weight loss method. Results showed that the inhibitor has excellent inhibition efficiency for corrosion of Q235 steel in neutral water medium; the inhibition efficiency increased with increase in inhibitor concentration, and then tend to be stable due to the inhibitors come to adsorption equilibrium in the metal surface. Using scanning electron microscope (SEM) to observe the micro corrosion morphology, and the corrosion mechanism is discussed in this article.
\end{abstract}

\section{Introduction}

With the rapid development of industrial technology, metal corrosion issue has become a global issue, corrosion involves a number of important areas of national production. According to statistics, every year the world economic loss due to corrosion caused by $3 \%$ $-5 \%$ of GDP, about $30 \%$ of the steel from corrosion caused a direct loss, other indirect loss is immeasurable. Such as boiler water, equipment and other neutral medium circulating cooling water systems used in pipeline etc facing serious corrosion problems ${ }^{[1]}$.

Corrosion inhibitors due to good effect and higher economic efficiency, has become one of the most widely used anti-corrosion technology approach. With the improvement of global environmental awareness, according to the sustainable development strategy, to promote green chemistry, good research and development performance, low-cost, non-toxic, pollution-free green corrosion inhibitor treatment is the development direction of ${ }^{[2-5]}$. In this paper, fatty acids and amino acids synthesized from two neutral media inhibitor (H1, $\mathrm{H} 2$ ), to study the performance of its carbon steel corrosion in the water system.

\footnotetext{
*Corresponding author: 164348096@qq.com
} 


\section{Experiment}

\subsection{Synthesis of inhibitor}

Using condensation method process route of Schotten-Baumann to synthesis target product with the raw material of fatty acid and amino acid ${ }^{[6-7]}$. The reaction equation is:
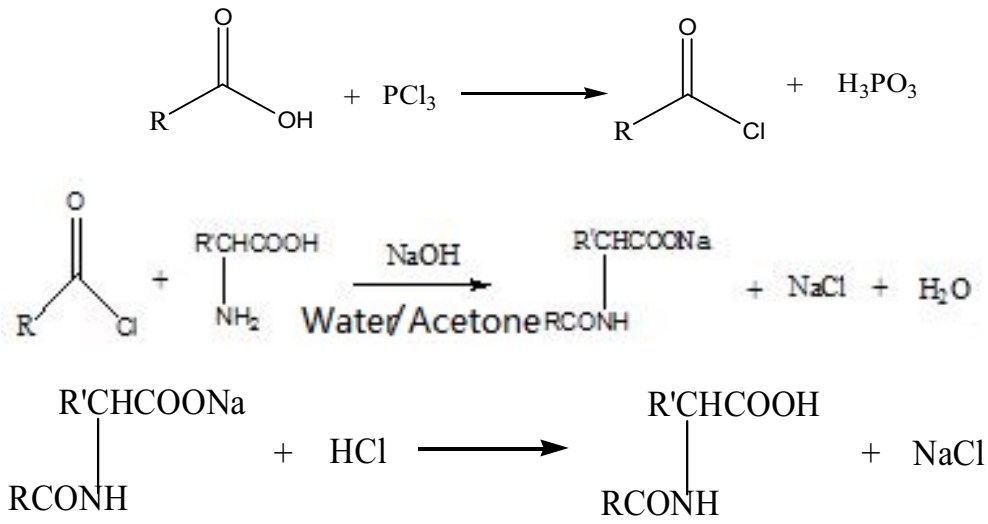

\section{Corrosion performance measurement}

\subsection{Weight-loss method}

According to the national standard, Select size of $76 \mathrm{~mm} \times 13 \mathrm{~mm} \times 1.5 \mathrm{~mm}$ of Q235 steel samples, weight-loss experiment with the test pieces after surface treatment and dry. Top water as corrosive medium, the experiment temperature of $303 \mathrm{~K}$, After 24 hours of immersion, take out pieces of steel, clean, dry, weighing, to work out the precise pieces of steel corrosion of weightlessness( $\mathrm{W} g$ ) before and after weight-loss. The inhibition efficiency was calculated according to the relation:

$$
V=\frac{W_{0}-W_{\mathrm{t}}}{\mathrm{s} * \mathrm{t}}
$$

Where $\mathrm{V}\left(\mathrm{g} /\left(\mathrm{m}^{2} \mathrm{~h}\right)\right)$ is the corrosion rate; $\mathrm{W}_{\mathrm{o}}(\mathrm{g})$ and $\mathrm{W}_{\mathrm{t}}(\mathrm{g})$ are respectively for the quality of the samples before and after weight-loss; $\mathrm{S}\left(\mathrm{m}^{2}\right)$ for specimens; $\mathrm{t}(\mathrm{h})$ for time.

$$
\eta=\frac{V_{0}-V_{\mathrm{t}}}{V_{0}} * 100 \%
$$

Where $\eta$ is the inhibition efficiency and $V_{o}\left(g /\left(m^{2} h\right)\right)$ and $V_{t}\left(g /\left(m^{2} h\right)\right)$ is the corrosion rate without and with inhibitor, respectively. 


\subsection{Corrosion morphology analysis}

The surface morphology of Q235 steel after weight-loss test is observed by QUANTA400 scanning electron microscopy.

\section{Results and Discussion}

\subsection{Weight-loss}

The corrosion static data of Q235 steel at $30{ }^{\circ} \mathrm{C}$ with adding different concentrations of inhibitors (H1, H2) in tap water medium were shown in Table 1 and Table 2. Figure 3 show the relationship of concentration and the inhibition efficiency.

Table 1 Corrosion Data Of H1

\begin{tabular}{|c|c|c|c|c|c|}
\hline $\mathrm{C}(\mathrm{mol} / \mathrm{L})$ & 0 & 100 & 150 & 200 & 300 \\
\hline $\mathrm{V}_{\text {corr }}(\mathrm{mm} / \mathrm{a})$ & 0.1277 & 0.0773 & 0.0225 & 0.0161 & 0.0140 \\
\hline$\eta(\%)$ & 0 & 39.5 & 82.4 & 87.4 & 89.0 \\
\hline
\end{tabular}

Table 2 Corrosion Data Of H2

\begin{tabular}{|c|c|c|c|c|c|}
\hline$C(\mathrm{~mol} / \mathrm{L})$ & 0 & 100 & 150 & 200 & 300 \\
\hline $\mathrm{V}_{\text {corr }}(\mathrm{mm} / \mathrm{a})$ & 0.1277 & 0.1062 & 0.0376 & 0.0333 & 0.0193 \\
\hline$\eta(\%)$ & 0 & 16.8 & 70.6 & 73.9 & 84.9 \\
\hline
\end{tabular}

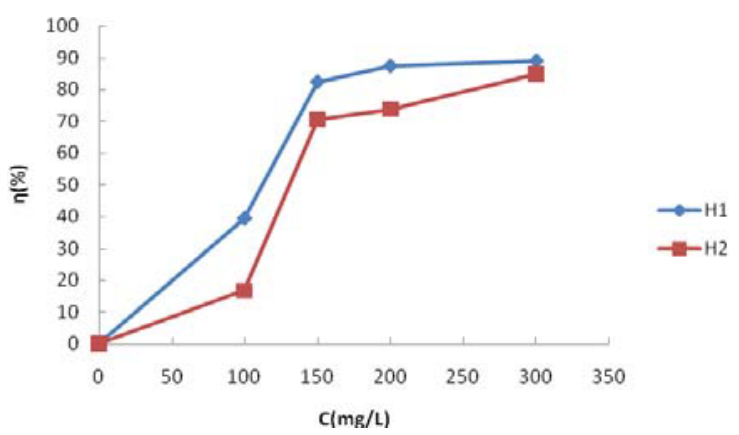

Figure 1 Inhibition efficiency of different concentrations of inhibitor at $30^{\circ} \mathrm{C}$

As it can be seen from Table 1, 2 and figure 3, the corrosion rate of Q235 steel were all reduced after adding inhibitor $\mathrm{H} 1$ and $\mathrm{H} 2$ with concentration of $150 \mathrm{mg} / \mathrm{L}$, two kinds of corrosion inhibitors exhibited good the inhibition effect. the inhibition efficiency increased with increase in inhibitor concentration. Then may be due to corrosion on the metal surface reason reach adsorption equilibrium, there will be stable inhibition efficiency phenomenon with the increases inhibitor concentration.

\subsection{Micro morphology of corrosion products}

Observing the mirco morphology of Q235 steel after adding different inhibitors(H1、H2) by scanning electron microscope. 


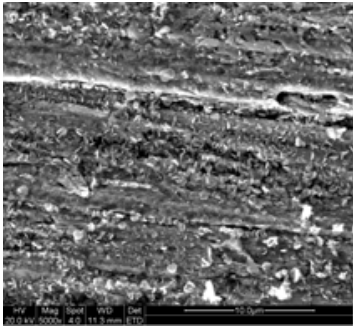

(a)

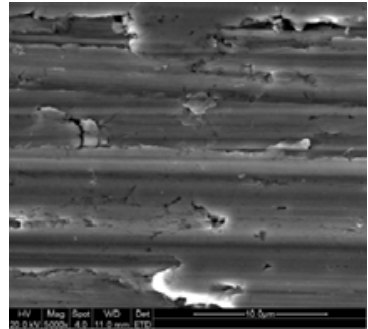

(b)

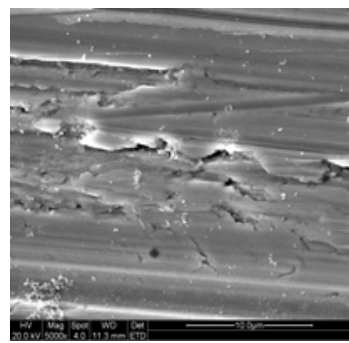

(c)

Fig. 2 SEM of Q235 steel in different inhibitor

Figure 2 is the surface topography of Q235 steel in the blank (a) and add inhibitor (b-H1、c-H2) water medium after a magnification of 5,000 times. The result shows that, there are lot of corrosion products and coarse cracks and pitting on the surface of Q235 steel in the blank water medium, the corrosion of Q235 steel is very serious; the corrosion is not so obvious after adding inhibitor, the sample surface is very bright, The results show that inhibitor(H1、H2) has a good inhibition effect on the corrosion of Q235 steel,and the inhibition efficiency of $\mathrm{H} 1$ is better than $\mathrm{H} 2$.

\subsection{Corrosion mechanism}

According to the analysis of experimental results of static weight loss method, Figure 3 is a schematic diagram of the adsorption model of inhibitor molecules in the anode surface.

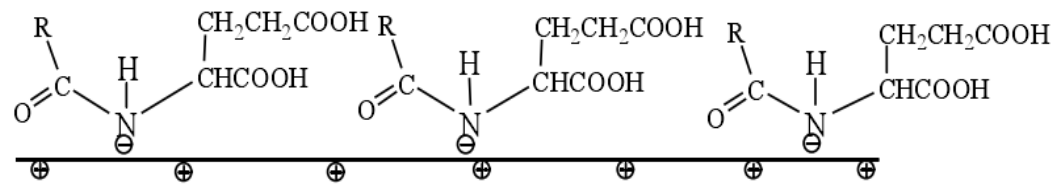

(H1)

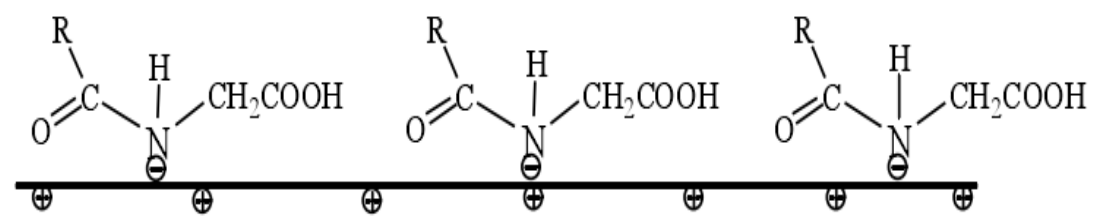

(H2)

Figure 3 adsorption model diagram of inhibitor in the anode surface

The figure shows, the nitrogen atom in the inhibitors molecule can better adsorption on the anode surface because of its strong electronegativity, between the metal / dielectric interface form a protective film, and thus played a very good metal inhibition effect. Weight loss method known from experimental data, the inhibition efficiency of $\mathrm{H} 1$ is higher than $\mathrm{H} 2$, the reason for this phenomenon may be due to $\mathrm{H} 1$ molecule contains more reactive groups $\left(-\mathrm{CH}_{2} \mathrm{CH}_{2} \mathrm{COOH}\right)$ thus lead to inhibition efficiency is better than $\mathrm{H} 2$.

\section{Conclusions}

Synthesis Inhibitor has good inhibition effect for Q235 steel in tap water, the two compounds added to water in a concentration of Q235 $150 \mathrm{mg} / \mathrm{L}$ that exhibited good 
corrosion effect, inhibition rate rises with increasing concentration of inhibitor, then may be due to corrosion on the metal surface reasons adsorption equilibrium is reached, there will be stable inhibition efficiency phenomenon with the increases inhibitor concentration. They have better prospects.

According to experimental data, studies suggest that inhibitor molecules due to their molecular nitrogen atom has a strong negative and thus can be adsorbed on the anode surface, at the metal / dielectric interface form a protective film, which play the role of electric corrosion. Under the same conditions, H1's inhibition efficiency is higher than $\mathrm{H} 2$, this phenomenon is due to the inhibition of nitrogen atoms in the molecule containing different substituents, it changed its adsorption capacity, and thus slow etchant inhibition efficiency impact.

\section{References}

1. Wang hui-long, zheng jia-shen. Progress of research on environmental-friendly corrosion inhibitions [J]. Corrosion science and protection technology, 2002,14(5): 275

2. Cheng peng, Fu chao-yang. Present State and Prospects of Biological Corrosion Inhibitors [J]. Total corrosion control,2011,25(9):11-15

3. Zheng jie, wang ya-na, zhang bo-linet al. research progress on natural products as corrosion inhibitor in acid,neural and alkaline mediums[J]. Corrosion science and protection technology,2011,23(1):103-106

4. Zhang Da-quan. Research Progress for Corrosion Inhibitor Science and Technology and Its Application in Energy Industry [J]. Journal of Shanghai University of Electric Power, 2013,29(4): 355-363

5. Cui R, Gu N, Li C. Polyaspartic acid as a green corrosion inhibitor for carbon steel[J]. Materials and Corrosion , 2011,62(4):362-369

6. Chen Yan-ni, Lu Yun, Wu Hao, et al. Progress on the preparation and application of $\mathrm{N}$-acylamino acids surfactants [J]. Chemical Research and Application, 2001, 13(2): 192-195

7. Zeng Ping, Xie Wei-yue. Progress on aynthesis and application of N-acylamino acid type surfactants[J]. Fine and Specialty Chemicals, 2008, 24(16): 13-17

8. Znini M,Cristofari G,Majidi L,et al. Green Approach to Corrosion Inhibition of Mild Steel by Essential Oil Leaves of Aslerisciis Graveo/ens(Forssk.) in Sulphuric Acid Medium[J].Int J Electrochem Sci, 2012, 7:3959-3981

9. Morad, MS. Effect of amino acids containing sulfur on the corrosion of mild steel in phosphoric acid solutions containing $\mathrm{Cl}^{-} 、 \mathrm{~F}^{-}$and $\mathrm{Fe}^{3+}$ ions: Behavior under polarization conditions[J]. Journal of applied electrochemistry, 2005, 35(9): 889 -895 